\title{
Dietary factors and lung function in the general population: wine and resveratrol intake
}

\author{
M. Siedlinski*, J.M.A. Boer" ${ }^{\#}$, H.A. Smit ${ }^{\top}$, D.S. Postma ${ }^{+}$and H.M. Boezen*
}

ABSTRACT: Wine intake is associated with a better lung function in the general population, yet the source of this effect is unknown. Resveratrol, a polyphenol in wine, has anti-inflammatory properties in the lung, its effects being partially mediated via induction of Sirtuin (SIRT)1 activity. We assessed the impact of wine and resveratrol intake, and SIRT1 single-nucleotide polymorphisms (SNPs) on lung function in the general population.

Effects of red and white wine and resveratrol intake on forced expiratory volume in $1 \mathrm{~s}$ (FEV1), forced vital capacity (FVC) and FEV1/FVC were analysed in the population-based Doetinchem cohort $(n=3,224)$. Associations of four tagging SIRT1 SNPs with lung function were analysed in the Doetinchem $(n=1,152)$ and Vlagtwedde-Vlaardingen $(n=1,390)$ cohorts.

Resveratrol intake was associated with higher FVC levels, and white wine intake with higher FEV1 levels and lower risk of airway obstruction. SIRT1 SNPs were not significantly associated with level or course of lung function, either directly or indirectly via wine or resveratrol intake.

This study shows a positive association of resveratrol intake with lung function in the general population, confirms the previously reported positive association of white wine intake with higher levels of FEV1, and additionally shows an association with a higher FEV1/FVC ratio. These effects probably do not run via SNPs in SIRT1.

KEYWORDS: Chronic obstructive pulmonary disease, lung function, resveratrol, single-nucleotide polymorphism, Sirtuin 1, wine

hronic obstructive pulmonary disease (COPD) is a worldwide-prevalent disease with increased mortality and morbidity [1]. An increased inflammatory process with an increased burden of proteases and oxidative stress contributes to progressive airway obstruction and COPD development $[2,3]$. The known contributors to lung function loss are of both environmental (e.g. smoking) and genetic origin [4-6].

Apart from smoking, other environmental factors are related to the level of lung function, including the consumption of several food compounds (e.g. vitamin C [7] and whole-grain products [8]). It has also been shown that out of several alcohol sources, only wine intake is positively associated with the level of forced expiratory volume in $1 \mathrm{~s}$ (FEV1) and forced vital capacity (FVC) in the general population [9], but the compound responsible for this effect remains unknown so far. A putative candidate that may account for the observed beneficial effect of wine is resveratrol, a polyphenol present in the skin of grapes. Resveratrol possesses antiinflammatory properties in airway epithelial cells [10] and alveolar macrophages derived from COPD patients [11]. The mechanism of this action may be explained by the resveratrol-mediated induction of Sirtuin (SIRT)1 activity [12, 13]. SIRT1 belongs to the class III histone/protein deacetylases.

The resveratrol-SIRT1 pathway contributes to lifespan prolongation in several organisms $[12,14]$ and improvement of mitochondrial function in mice $[15,16]$. It has recently been shown that cigarette smoke impairs SIRT1 function in human macrophages in vitro and rat lungs in vivo, which resulted in an increase of nuclear factor- $\kappa \mathrm{B}-$ dependent inflammatory burden [13]. Interestingly, this effect was inhibited by resveratrol. Furthermore, the observed post-translational modifications of SIRT1 protein by cigarette smoke explained the lower SIRT1 expression in lung tissue of healthy smokers and COPD patients, as compared with neversmokers [17].

\section{AFFILIATIONS}

*Depts of Epidemiology,

+Pulmonology, University Medical Center Groningen, University of Groningen, Groningen,

${ }^{\#}$ National Institute for Public Health and the Environment, Bilthoven, and - Julius Center for Health Sciences and Primary Care, University Medical Center Utrecht, Utrecht, The Netherlands.

CORRESPONDENCE

H.M. Boezen

Dept of Epidemiology, E3.29

University Medical Center Groningen P0 Box 30.001

9700 RB Groningen

The Netherlands

E-mail: h.m.boezen@epi.umcg.nl

Received

Nov 302010

Accepted after revision:

July 012011

First published online:

Aug 182011 
In the current study, performed in the large $(n=3,224)$, prospective population-based Doetinchem cohort, we tried to replicate the previously reported positive association between wine intake and the level of lung function [9]. Furthermore, we calculated total resveratrol intake and assessed its association with the level and longitudinal decline of lung function that, if abnormally accelerated, can result in the development of COPD [18]. Additionally, we studied the role of SIRT1 genetic variations and their interaction with smoking in relation to lung function in two distinct, prospective cohorts, i.e. random samples of the Doetinchem and Vlagtwedde-Vlaardingen cohorts in the Netherlands.

\section{METHODS}

\section{Subjects}

The associations between lung function and intake of food compounds and resveratrol intake were assessed in the Doetinchem cohort study [19], a prospective part of the larger MORGEN (Monitoring Project on Risk Factors and Health in the Netherlands) study [7]. This Caucasian cohort consisted of 3,224 subjects with DNA and spirometry tests available (table 1). We selected a random subsample for DNA analysis $(n=1,152$; table E1 in the online supplementary material), as described previously [20]. Between 1994 and 2007, subjects were tested for pre-bronchodilator lung function (FEV1 and FVC) three times at 5-yr intervals according to the European Respiratory Society (ERS) guidelines [21]. Similarly to the entire Doetinchem cohort, in the random subsample, $100 \%, 100 \%$ and $70.4 \%$ of subjects participated in the first, second and third surveys, respectively. The characteristics of the subjects who were lost to follow-up were not significantly different from those who were examined at the third survey.

The Vlagtwedde-Vlaardingen cohort $(n=1,390$; previously described in detail [22]) was included to replicate genetic findings of the Doetinchem cohort (table E1 in the online supplementary material). This Caucasian cohort was prospectively followed for 25 yrs with lung function measurements every 3 yrs. See the online supplementary material for details on lung function measurements in the Doetinchem and Vlagtwedde-Vlaardingen cohorts.

In the Vlagtwedde-Vlaardingen cohort, FEV1 measurements were included from the age of $30 \mathrm{yrs}$, because an individual's maximally attained lung function is assumed to have been reached before that age and lung function is considered to be either in the plateau or decline phase [22]. In the Doetinchem cohort, all subjects had passed the age of 30 yrs at the second survey and, thus, were included in the analysis.

There was no overlap in subjects whatsoever between the two cohorts, due to a different sampling time frame and sampling from different cities under the responsibility of different institutes. The study protocols were approved by local medical ethics committees and all participants gave their written informed consent.

\section{Assessment of the dietary intake in the Doetinchem cohort}

Dietary intake of 178 food items was assessed using a semiquantitative food frequency questionnaire filled out by all participants prior to the first and second surveys in the Doetinchem cohort $(n=3,224)$ [19]. In order to better estimate the

\begin{tabular}{|c|c|c|}
\hline TABLE 1 & \multicolumn{2}{|c|}{$\begin{array}{l}\text { Characteristics of the total Doetinchem cohort at } \\
\text { the second survey }\end{array}$} \\
\hline Time of visi & & 1999-2002 \\
\hline Subjects n & & 3224 \\
\hline Males & & $1560(48.4)$ \\
\hline Age yrs & & $50.0(31.2-70.9)$ \\
\hline Height $\mathrm{cm}$ & & $172.8 \pm 9.2$ \\
\hline Smokers/ex & mokers & $859(26.7) / 1259(39.1)$ \\
\hline \multicolumn{3}{|c|}{ Smoking exposure pack-yrs } \\
\hline Total coho & & $4.6(0.0-154.0)$ \\
\hline Ever-smok & & $13.0(0.004-154.0)$ \\
\hline FEV1 L & & $3.41 \pm 0.80$ \\
\hline FVC L & & $4.38 \pm 1.02$ \\
\hline FEV 1 chang & $\mathrm{mL} \cdot \mathrm{yr}^{-1}$ & $-26.03 \pm 34.07$ \\
\hline \multicolumn{3}{|c|}{ Consumers $\%$, median (IQR) in consumers } \\
\hline Wine $g \cdot d a$ & & $76.2,23.3(7.1-67.6)$ \\
\hline White & & $65.1,7.4(2.5-21.4)$ \\
\hline Red & & $65.4,15.4(3.3-50.0)$ \\
\hline Grapes $g \cdot c$ & & $99.4,8.1(4.3-13.9)$ \\
\hline Resveratro & Ig. day ${ }^{-1}$ & 100.0, $122.9(36.0-506.2)$ \\
\hline From no & grape products & $100.0,8.0(5.6-11.6)$ \\
\hline \multicolumn{3}{|c|}{$\begin{array}{l}\text { Food item \% contribution to resveratrol intake } \\
\text { (resveratrol concentration mg per } 100 \mathrm{~g} \text { ) }\end{array}$} \\
\hline Red wine & & $84.1(1.43)$ \\
\hline White wine & & $9.5(0.33)$ \\
\hline Grapes & & $4.3(0.20)$ \\
\hline Raw tomat & & $0.6(0.06)$ \\
\hline Peanut but & & $0.4(0.07)$ \\
\hline Chocolate & & $0.3(0.05)$ \\
\hline Strawberrie & & $0.2(0.02)$ \\
\hline Chocolate & & $0.2(0.02)$ \\
\hline Peanuts & & $0.1(0.01)$ \\
\hline Peanut sal & & $0.1(0.04)$ \\
\hline Chocolate & & $0.1(0.002)$ \\
\hline
\end{tabular}

Data are presented as $\mathrm{n}(\%)$, median (range) or mean $\pm \mathrm{SD}$, unless otherwise stated. FEV1: forced expiratory volume in $1 \mathrm{~s}$; FVC: forced vital capacity; IQR: interquartile range. ${ }^{\#}$ : calculated with the last and the first available FEV1 measurements; $\because$ includes the resveratrol intake derived from tomatoes, strawberries, and cocoa- and peanut-containing food items.

lifetime consumption of these products, the average intake from both surveys was calculated and used in all analyses. Resveratrol intake was calculated using the previously reported resveratrol content in 11 food items (table 1; see online supplementary material for details) [23-27].

\section{SIRT1 genotyping}

Four single-nucleotide polymorphisms (SNPs), which tag all 22 SNPs in SIRT1 and its 5-kb up-/downstream region with $\mathrm{r}^{2}<0.8$ and a minor allele frequency $>5 \%$ (based on the HapMap release 23a, March 2008), i.e. rs12778366, rs10823108, rs7069102 and rs2273773, were genotyped (table 2). Since SNPs rs10823108 and rs2273773 were in complete linkage disequilibrium $\left(r^{2}=1.0\right)$ in both cohorts, only data on rs2273773 are presented. Four haplotypes in SIRT1 were identified and only one (containing the mutant allele of rs7069102 and wild-type alleles of all the other SNPs) was unique, i.e. was not tagged by a single allele of 
TABLE 2 Sirtuin (SIRT)1 genotype characteristics in the two cohorts studied

\begin{tabular}{|c|c|c|c|c|c|c|c|c|}
\hline \multirow[t]{2}{*}{ SIRT1 variation } & \multicolumn{4}{|c|}{ Doetinchem cohort ${ }^{\#}$} & \multicolumn{4}{|c|}{ Vlagtwedde-Vlaardingen cohort } \\
\hline & Heterozygotes & $\begin{array}{c}\text { Homozygous } \\
\text { mutants }\end{array}$ & MAF \% & $\begin{array}{l}\text { HWE test } \\
\text { p-value }\end{array}$ & Heterozygotes & $\begin{array}{c}\text { Homozygous } \\
\text { mutants }\end{array}$ & MAF \% & $\begin{array}{l}\text { HWE test } p- \\
\text { value }^{+}\end{array}$ \\
\hline rs12778366 ${ }^{\S}$ & $264(23.2)$ & $27(2.4)$ & 13.9 & 0.23 & $290(21.5)$ & $14(1.0)$ & 11.8 & 0.21 \\
\hline rs7069102 ${ }^{f}$ & $529(46.9)$ & $145(12.8)$ & 36.3 & 0.65 & $576(43.8)$ & $153(11.6)$ & 33.5 & 0.52 \\
\hline
\end{tabular}

Data are presented as $n(\%)$, unless otherwise stated. MAF: minor allele frequency; HWE: Hardy-Weinberg equilibrium. ${ }^{*}: n=1,152 ;$ total SIRT1 single-nucleotide polymorphism (SNP) call rate $98.4 \%{ }^{\circ}: \mathrm{n}=1,390$; total SIRT1 SNP call rate $96.4 \%{ }^{+}$: for one degree of freedom Chi-squared test comparing the observed genotype

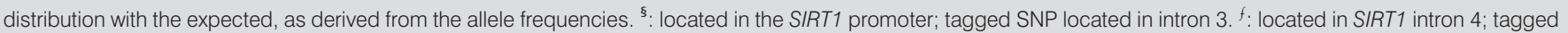
SNPs located in the promoter, introns $1-8$ and $3^{\prime}$-proximal region. ${ }^{\# \#}$ : synonymous SNP (Leu332Leu) in exon 5; tagged SNPs located in the promoter and introns 3-5.

any investigated SIRT1 SNP (see online supplementary material and table E4 for details).

\section{Statistics}

Linear regression was performed to assess associations of the continuously defined average intake of total resveratrol, wine intake, grapes and resveratrol from nongrape products with the level of FEV1 and FVC at the second survey in the Doetinchem cohort $(n=3,224$; table 1$)$. The average intake of all investigated dietary compounds was $\log _{10}$-transformed to obtain normal distributions $\left(\log _{10}(\right.$ intake +1$)$ for the intake of wine and grapes). Identified significant associations were further analysed as tertiles and presented in plots.

Logistic regression was used to analyse the associations between the continuously defined average intake of wine and total resveratrol intake, and the prevalence of airway obstruction as

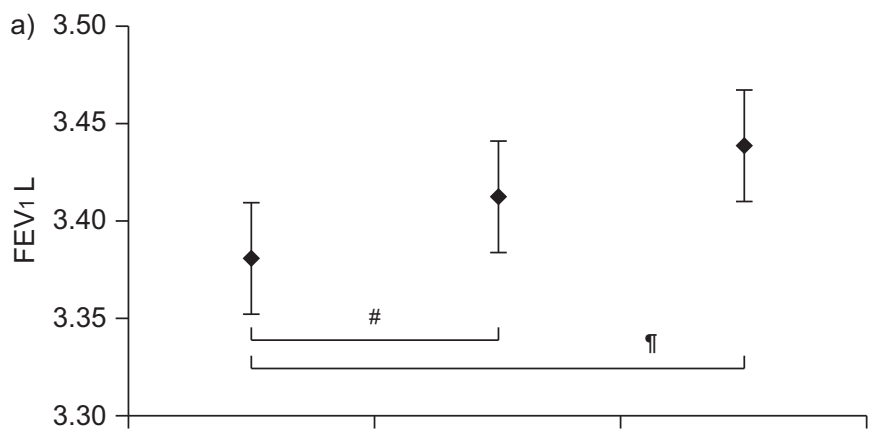

b)
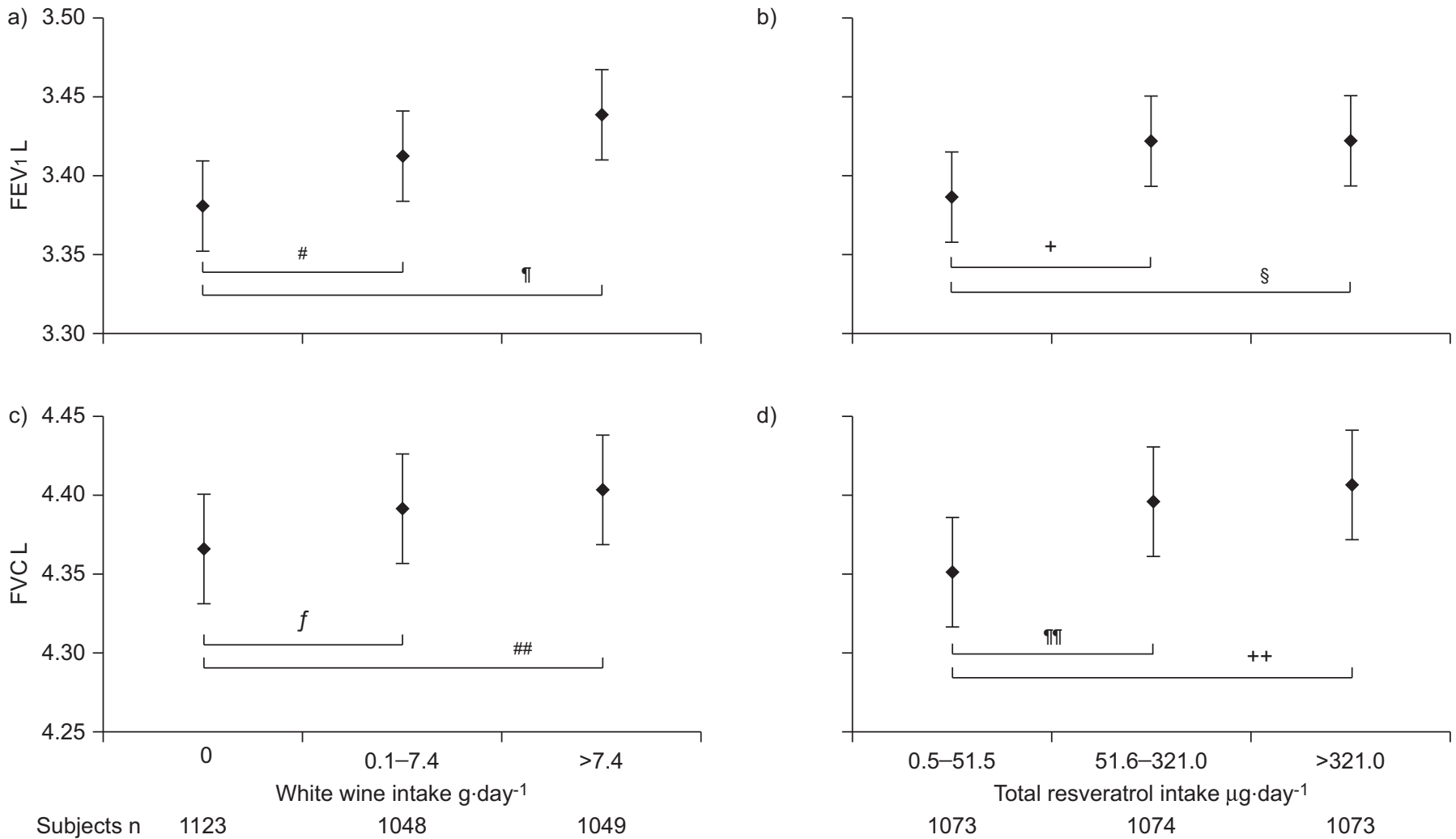

d)

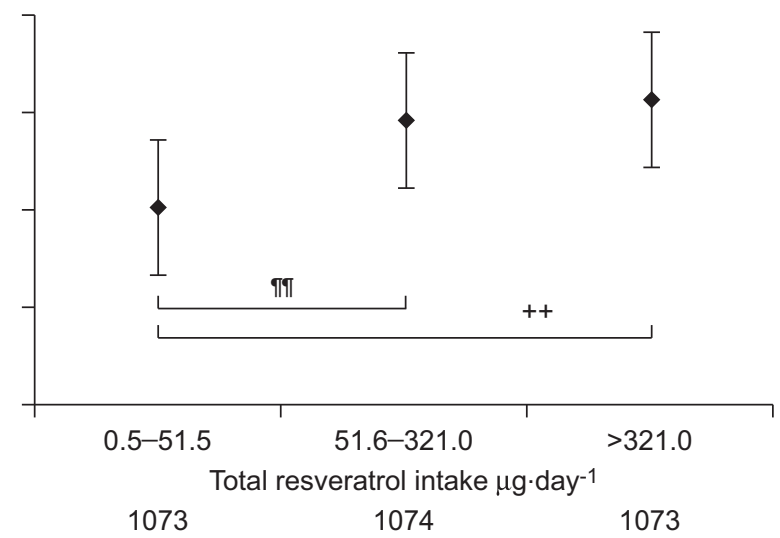

FIGURE 1. Mean adjusted $\mathrm{a}, \mathrm{b})$ forced expiratory volume in $1 \mathrm{~s}(\mathrm{FEV} 1)$ and $\mathrm{c}, \mathrm{d})$ forced vital capacity $(\mathrm{FVC})$ for the subjects according to the average intake of a, $c)$ white wine and $b, d)$ total resveratrol in the Doetinchem cohort $(n=3,224)$. Data are presented as mean $(95 \% \mathrm{Cl})$. White wine intake is presented according to the intake status and a median intake of $7.4 \mathrm{~g} \cdot$ day $^{-1}$ in white wine consumers. Total resveratrol intake is presented in tertiles. ${ }^{\#}: p=0.12 ;{ }^{\bullet}: p=0.01 ;{ }^{+}: p=0.08 ;{ }^{5}: p=0.11 ;{ }^{f}: p=0.30 ;{ }^{\# \#}: p=0.15$; II: $p=0.02 ;{ }^{++}: p=0.03$. 

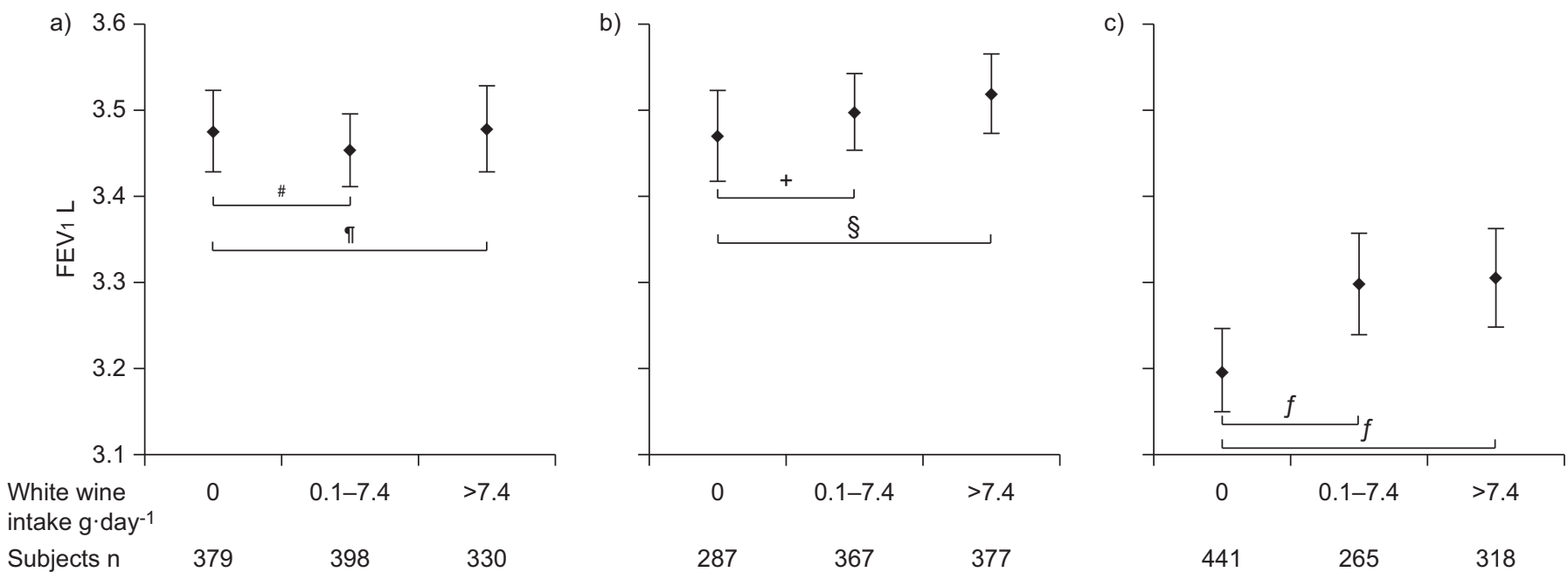

FIGURE 2. Mean adjusted forced expiratory volume in $1 \mathrm{~s}(\mathrm{FEV} 1)$ according to the average intake of white wine and pack-years smoked in the Doetinchem cohort $(n=3,224)$. a) Never-smokers, b) mild smokers ( $\leqslant 13$ pack-yrs) and c) heavy smokers ( $>13$ pack-yrs). Data are presented as mean $(95 \% \mathrm{Cl})$. White wine intake is presented according to the intake status and a median intake of $7.4 \mathrm{~g} \cdot$ day $^{-1}$ in white wine consumers. ${ }^{\#}: p=0.50 ;{ }^{\top}: p=0.95 ;{ }^{+}: p=0.43 ;{ }^{\S}: p=0.21 ;{ }^{f}: p=0.01$.

defined by the lower limit of normal (LLN) FEV1/FVC ratio and LLN FEV1, at the second survey (see online supplementary material for the definition of LLN).

Analyses were performed either using SPSS, version 14.0 (regression analyses; IBM, Amsterdam, the Netherlands) or S-PLUS, version 7.0 (linear mixed-effects models; Tibco, Gothenburg, Sweden). See the online supplementary material for details on the statistical analyses. A p-value of $<0.05$ was considered to be statistically significant (tested two-sided).

\section{RESULTS}

\section{FEV1, FVC and airway obstruction in relation to wine and resveratrol intake in the Doetinchem cohort}

All subjects consumed resveratrol in some form and red wine was the predominant contributor to the overall resveratrol intake (table 1).

We found no association between red wine intake and FEV1, FVC or airway obstruction. The intake of white wine was associated with higher FEV1 level, whereas the intake of resveratrol was associated with higher FVC level (fig. 1 and supplementary table E2).

Since smoking may reduce the anti-inflammatory capacity of SIRT1, an effect inhibited by resveratrol, we further analysed white wine and resveratrol intake for their interaction with pack-years smoked. There was a significant interaction of pack-years smoked and white wine intake with the FEV1 $(p=0.03)$, and a nonsignificant interaction with FVC ( $p=0.07)$. This interaction reflected an association between white wine consumption and higher FEV1 in heavy smokers only (defined using median of pack-years smoked in ever-smokers; fig. 2). The interaction term between total resveratrol intake and pack-years smoked was not significant with respect to FEV1 $(p=0.17)$ or FVC $(p=0.27)$.

White wine intake was significantly associated with a decreased risk of airway obstruction, defined as FEV1/FVC $<$ LLN and FEV1 $<$ LLN (table E2 in the online supplementary material). Lack of white wine consumption significantly $(p=0.017)$ increased the risk for the combination of FEV1 $<\mathrm{LLN}$ and FEV1/FVC $<\mathrm{LLN}$, a sign of COPD (fig. 3).

\section{FEV1 decline in relation to wine and resveratrol intake in the Doetinchem cohort}

There was no significant association between the intake of resveratrol or red or white wine and the change in FEV1 across the three surveys in the Doetinchem cohort.

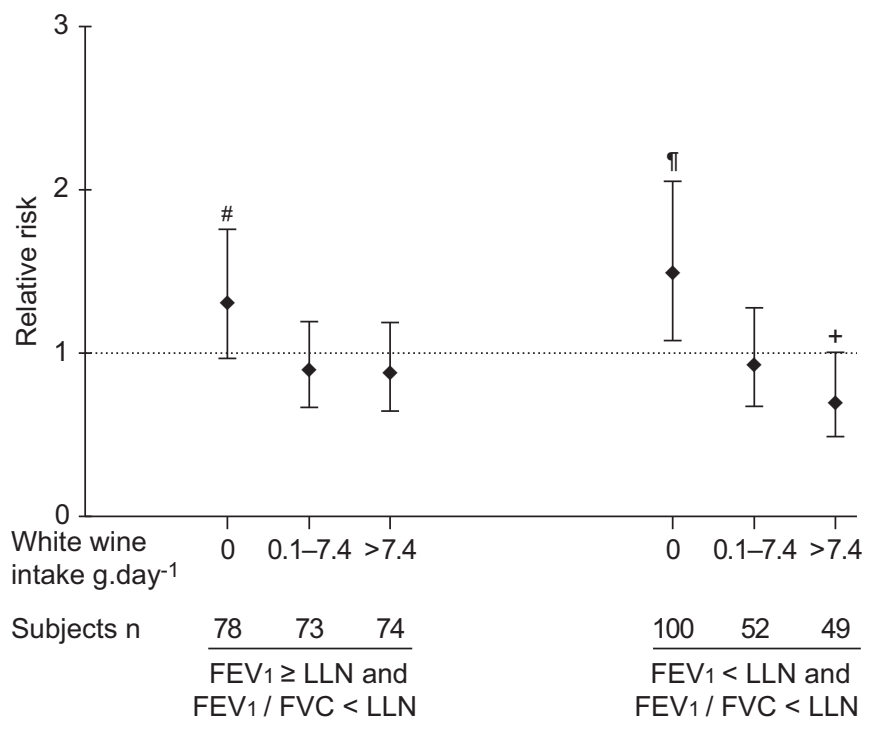

FIGURE 3. Adjusted relative risks for a low forced expiratory volume in $1 \mathrm{~s}$ (FEV1)/forced vital capacity (FVC) ratio and normal FEV1, and for a low FEV1/FVC and low FEV 1 according to the intake of white wine. Data are presented as relative risk $(95 \% \mathrm{Cl})$. The reference group $(\mathrm{n}=2,290)$ had $F E V_{1} / F V C \geqslant$ lower limit of normal (LLN) and FEV $1 \geqslant L L N$, and no respiratory symptoms (breathlessness, chronic cough or chronic phlegm production). Numbers correspond to subjects identified within each group in the Doetinchem cohort. ${ }^{\#}: p=0.089 ;{ }^{\circ}: p=0.017 ;{ }^{+}: p=0.051$. 


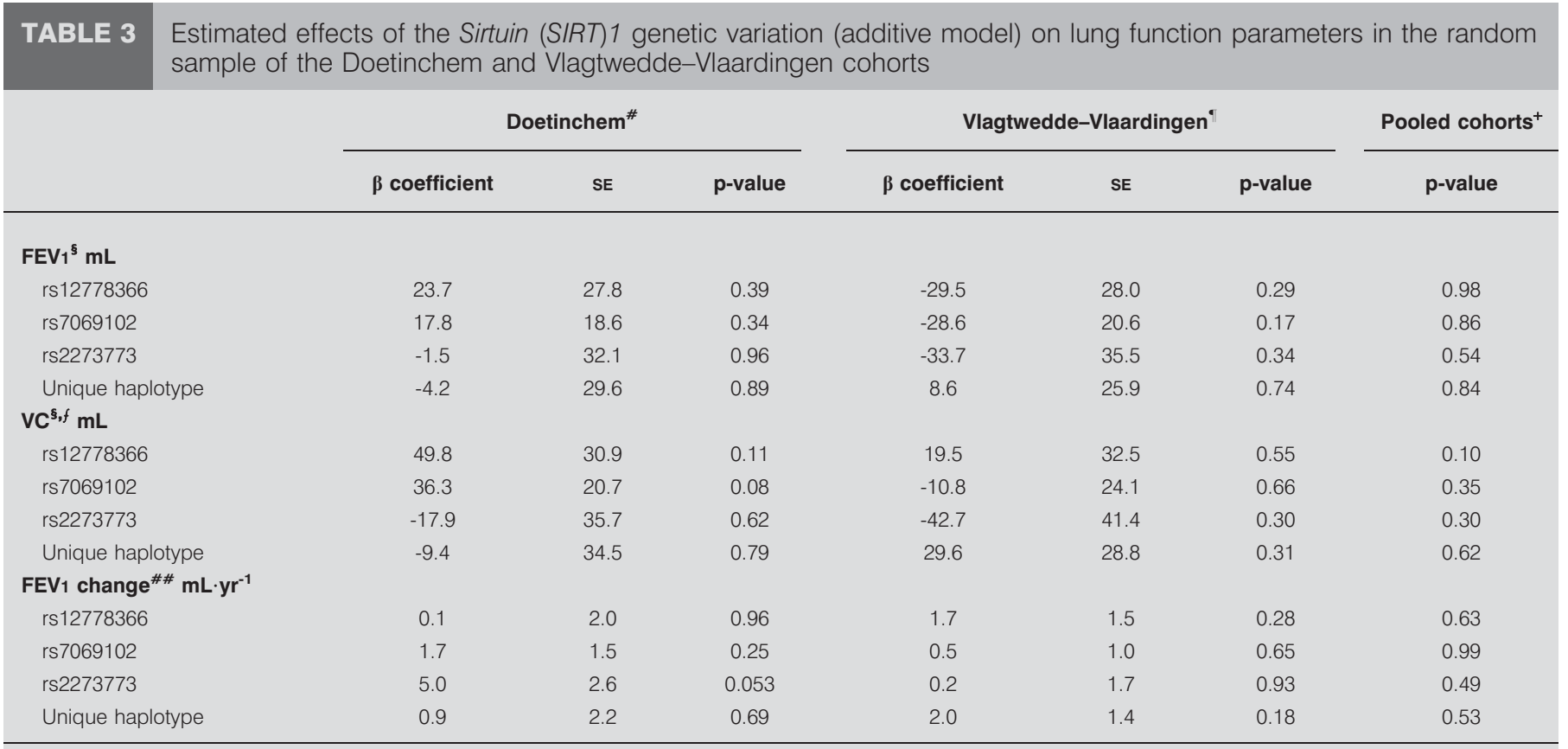

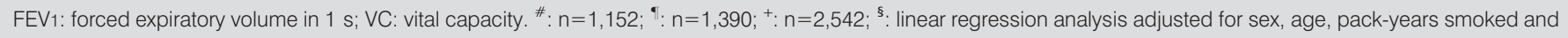
height, and the cohort binary variable for the pooled analysis; ${ }^{f}$ : measured as forced VC in the Doetinchem cohort and inspiratory VC in the Vlagtwedde-Vlaardingen

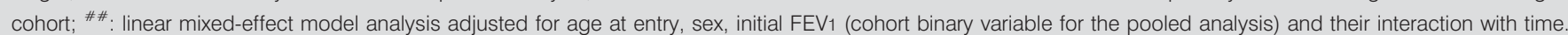

\section{Genetic variations in SIRT1, and lung function level and decline in the Doetinchem cohort and Vlagtwedde- Vlaardingen cohorts}

None of the SIRT1 genetic variations was significantly associated with FEV1 or FVC in an additive model (table 3). Additionally, in the pooled cohort, none of the SIRT1 variations was associated with FEV1, FVC, vital capacity (VC), or FEV1 or VC decline when analysed with the recessive or dominant model (data not shown).

Three significant interactions between SIRT1 genetic variations (additive model) and pack-years smoked were observed in the Doetinchem cohort and one other in the VlagtweddeVlaardingen cohort in relation to FEV1 and FVC, but none of these was observed in the pooled cohort analysis (table E4 in the online supplementary material).

We found no significant interaction between SIRT1 variations and resveratrol intake with respect to FEV1 or FVC at the second survey in the random sample of the Doetinchem cohort in any genetic model (see table E5 in the online supplementary material for the additive model).

\section{DISCUSSION}

To our knowledge, this is the first study investigating resveratrol intake in relation to level and progressive loss of lung function. We found a positive association of resveratrol intake with FVC level in the general population. Furthermore, we replicated the previously observed positive association of white wine intake with FEV1 in the general population [9], and extended this observation by showing that white wine intake is positively associated with a lower risk of an abnormally low FEV1/FVC ratio, a measure of airway obstruction, as present in COPD. Our results do not imply that heavy wine consumption is protective in any respect. Finally, we conclude that polymorphisms in SIRT1 do not appear to affect lung function either via an independent association, or via interaction with pack-years smoked or the intake of resveratrol.

Beneficial effects of wine consumption are postulated to account for the "French paradox", the observation of lower mortality due to coronary heart disease in the French population despite this population's relatively high consumption of a cholesterol- and saturated fat-rich diet $[28,29]$. The proposed mechanism includes the inhibition of platelet reactivity by wine [28] and resveratrol is a prominent candidate responsible for the vascular protection provided by wine [30]. However, our study shows that white wine intake and not red wine intake, the major dietary resveratrol source, is associated with a lower risk of airway obstruction and with higher FEV1, both of which are indices of COPD. This is of great importance given the fact that reduced lung function is a marker for cardiovascular-related mortality [31].

While we found that resveratrol intake was associated with a higher FVC, its association with FEV1 was not significant. Similarly, we did not observe significant associations of resveratrol intake with the presence of airway obstruction. Therefore, the relevance of the observed association in relation to respiratory disorders other than COPD needs to be further addressed. Interestingly, none of the separate grape sources of resveratrol was associated with FVC and neither was the resveratrol from nongrape sources (data not shown), which suggests that the overall effect of resveratrol intake on FVC is specific and independent of the source (table E2 in the online supplementary material).

The analysis of individual sources of resveratrol revealed that white wine intake is particularly associated with a higher FEV1. Accordingly, SCHÜNEMANN et al. [9] found white wine to display a 
stronger effect on FEV1 level compared with red wine in the cohort of 1,555 New York state (USA) residents. They suggested that health-promoting lifestyle factors associated with white wine drinking might be responsible for this effect. We would like to put forward that other polyphenolic compounds present in white wine may exert beneficial effects on lung function as well. Plausible candidates in this respect are tyrosol and hydroxytyrosol, polyphenolic white wine molecules that, similar to resveratrol, exhibit antioxidant and cardioprotective effects in vivo [32]. Other candidates are flavonoids that were associated with a higher FEV1 in the MORGEN study [33]. However, since flavonoids, such as quercetin and myricetin, are predominantly present in red wine [34], they are probably not responsible for the positive associations with white wine that we found.

We additionally showed that the intake of white wine interacts with the number of cigarettes smoked. Smokers particularly benefited from a higher white wine intake. This observation suggests that white wine might be efficient in the detoxification of molecules derived from cigarette smoke that elevate the oxidative or inflammatory burden in the lung and, thus, the positive associations of white wine intake with lung function become more easily apparent in smokers as compared with never-smokers. Finally, as shown in our study, nondrinkers of white wine are at increased risk of abnormally low FEV1 and FEV1/FVC levels, both of which are indices of COPD in a moderate stage.

We cannot rule out the possibility that the associations we found between white wine and lung function might be due to confounding, as the habit of drinking white wine might be associated with beneficial characteristics that are actually underlying the relationship. However, adjustments for factors we know to be associated with better lung function (e.g. higher socioeconomic status and other health beneficial factors) did not substantially change the effect estimates. Reverse causation might be an issue related to this, as people with better health and better lung function might be more affluent and be more likely to drink white wine. Overall, a causal relationship between white wine drinking and lung function has not been established, and it would be premature to make clinical recommendations based solely on the current results.

Additionally, we feel it is important to stress that the positive association we observed between white wine intake and higher FEV1 reflects a very modest average consumption of 1.0-1.5 glasses of white wine per week (i.e. $7.4 \mathrm{~g} \cdot \mathrm{day}^{-1}$ ) and, thus, we by no means aim to suggest that more than moderate white wine drinking could be considered as beneficial health behaviour.

We could not include all known dietary intakes of resveratrol, i.e. grape juice and fortified wine or other sources suggested to contain resveratrol, such as hops, which are present in beer [35], or cherries [36]. Nevertheless, our estimation of resveratrol intake might be acknowledged as sensitive on a population level, as the sources that were not used contain a low concentration of resveratrol $[27,35,36]$ and/or are not widely consumed in the total population.

The reproducibility and validity of the food frequency questionnaire used has been well documented in the literature $[37,38]$. The reproducibility of alcohol intake and the consumption of alcoholic beverages at 6 and 12 months was high (Spearman's rank correlation $0.83-0.94)$, as was the relative validity against 12monthly 24-h recalls (0.74-0.94). Therefore, we expect the reproducibility and relative validity of resveratrol intake and wine consumption to be high as well.

The sensitivity of the current estimation of the resveratrol intake is certainly lower on an individual level. This is due to the fact that the resveratrol content of grapes and wines is affected by many factors that could not be taken into account in the current study. In particular, it has been shown that the resveratrol content of wine depends on the type of wine and the region where the grapes were harvested, due to the climate/species-dependent resveratrol synthesis by grapes [23, 39, 40].

Since resveratrol induces deacetylase SIRT1 activity in vitro [1214] and SIRT1 expression is decreased in lung tissue of COPD patients [17], we were interested in analysing genetic variations in this gene in the context of lung function. Although we found some significant associations of lung function with SIRT1 SNPs and their interaction with smoking, none of these associations was replicated in the two cohorts investigated. The current study also did not identify any significant interaction between SIRT1 SNPs and resveratrol intake with respect to the level of lung function.

In summary, we demonstrate positive association of white wine intake and higher levels of FEV1 in the general population, which is pronounced in smokers. Consequently, white wine consumption is associated with a lower risk of moderate airway obstruction. We show, for the first time, that resveratrol intake is associated with higher FVC on a general population level, which needs further study. Moreover, genetic variants in SIRT1, a deacetylase gene that can be induced by resveratrol in vivo, are unlikely to play a role in the positive association between resveratrol and lung function.

\section{SUPPORT STATEMENT}

This research was funded by the Graduate School for Drug Exploration (University Medical Center Groningen, University of Groningen, Groningen, the Netherlands) and the Netherlands Asthma Foundation (grant NAF3.2.02.51).

\section{STATEMENT OF INTEREST}

None declared.

\section{ACKNOWLEDGEMENTS}

The authors thank the epidemiologists and fieldworkers of the Municipal Health Services in Doetinchem (the Netherlands) for their important contribution to the data collection of the Doetinchem Study, as well as J. Seidell, M. Verschuren and B. Bueno-de Mesquita (all National Institute of Public Health and the Environment (RIVM), Bilthoven, the Netherlands) for conducting the study, and A. Blokstra and P. Vissink (both Centre for Prevention and Health Services Research, RIVM) for the logistic and data management. We thank J. Schouten (Dept of Epidemiology, University Medical Center Groningen, University of Groningen, Groningen, the Netherlands) for the continuous management of the VlagtweddeVlaardingen data. Last but not least, the authors thank the participants of the Doetinchem and Vlagtwedde-Vlaardingen studies for their loyal participation in every survey.

\section{REFERENCES}

1 Gold Initiative for Chronic Obstructive Lung Disease. Global Strategy for the Diagnosis, Management and Prevention of COPD. 
www.goldcopd.org/Guidelines/guidelines-global-strategy-fordiagnosis-management-2007.html Date last updated: 2007.

2 Kirkham P, Rahman I. Oxidative stress in asthma and COPD: antioxidants as a therapeutic strategy. Pharmacol Ther 2006; 111: 476-494.

3 MacNee W. Pulmonary and systemic oxidant/antioxidant imbalance in chronic obstructive pulmonary disease. Proc Am Thorac Soc 2005; 2: 50-60.

4 The Alpha-1-Antitrypsin Deficiency Registry Study Group. Survival and FEV1 decline in individuals with severe deficiency of $\alpha_{1}$-antitrypsin. Am J Respir Crit Care Med 1998; 158: 49-59.

5 Redline S, Tishler PV, Rosner B, et al. Genotypic and phenotypic similarities in pulmonary function among family members of adult monozygotic and dizygotic twins. Am J Epidemiol 1989; 129: 827-836.

6 Kueppers F, Miller RD, Gordon H, et al. Familial prevalence of chronic obstructive pulmonary disease in a matched pair study. Am J Med 1977; 63: 336-342.

7 Grievink L, Smit HA, Ocke MC, et al. Dietary intake of antioxidant (pro)-vitamins, respiratory symptoms and pulmonary function: the MORGEN study. Thorax 1998; 53: 166-171.

8 Tabak C, Smit HA, Heederik D, et al. Diet and chronic obstructive pulmonary disease: independent beneficial effects of fruits, whole grains, and alcohol (the MORGEN study). Clin Exp Allergy 2001; 31: 747-755.

9 Schünemann HJ, Grant BJ, Freudenheim JL, et al. Beverage specific alcohol intake in a population-based study: evidence for a positive association between pulmonary function and wine intake. $B M C$ Pulm Med 2002; 2: 3.

10 Donnelly LE, Newton R, Kennedy GE, et al. Anti-inflammatory effects of resveratrol in lung epithelial cells: molecular mechanisms. Am J Physiol Lung Cell Mol Physiol 2004; 287: L774-L783.

11 Culpitt SV, Rogers DF, Fenwick PS, et al. Inhibition by red wine extract, resveratrol, of cytokine release by alveolar macrophages in COPD. Thorax 2003; 58: 942-946.

12 Howitz KT, Bitterman KJ, Cohen HY, et al. Small molecule activators of sirtuins extend Saccharomyces cerevisiae lifespan. Nature 2003; 425: 191-196.

13 Yang SR, Wright J, Bauter M, et al. Sirtuin regulates cigarette smoke-induced proinflammatory mediator release via RelA/p65 $\mathrm{NF}-\mathrm{KB}$ in macrophages in vitro and in rat lungs in vivo: implications for chronic inflammation and aging. Am Physiol Lung Cell Mol Physiol 2007; 292: L567-L576.

14 Wood JG, Rogina B, Lavu S, et al. Sirtuin activators mimic caloric restriction and delay ageing in metazoans. Nature 2004; 430: 686-689.

15 Lagouge M, Argmann C, Gerhart-Hines Z, et al. Resveratrol improves mitochondrial function and protects against metabolic disease by activating SIRT1 and PGC-1 $\alpha$. Cell 2006; 127: 1109-1122.

16 Koo SH, Montminy M. In vino veritas: a tale of two sirt1s? Cell 2006; 127: 1091-1093.

17 Rajendrasozhan S, Yang SR, Kinnula VL, et al. SIRT1, an inflammatory and antiaging protein is decreased in lungs of patients with chronic obstructive pulmonary disease. Am J Respir Crit Care Med 2008; 177: 861-870.

18 Antó JM, Vermeire P, Vestbo J, et al. Epidemiology of chronic obstructive pulmonary disease. Eur Respir J 2001; 17: 982-994.

19 Verschuren WM, Blokstra A, Picavet HS, et al. Cohort profile: the Doetinchem Cohort Study. Int J Epidemiol 2008; 37: 1236-1241.

20 Siedlinski M, Postma DS, van Diemen CC, et al. Lung function loss, smoking, vitamin C intake, and polymorphisms of the glutamatecysteine ligase genes. Am J Respir Crit Care Med 2008; 178: 13-19.

21 Quanjer PH, Tammeling GJ, Cotes JE, et al. Lung volumes and forced ventilatory flows. Report Working Party Standardization of Lung Function Tests, European Community for Steel and Coal. Official Statement of the European Respiratory Society. Eur Respir J 1993; 6: Suppl. 16, 5-40.
22 van Diemen CC, Postma DS, Vonk JM, et al. A disintegrin and metalloprotease 33 polymorphisms and lung function decline in the general population. Am J Respir Crit Care Med 2005; 172: 329-333.

23 Ribeiro de Lima MT, Waffo-Téguo P, Teissedre PL, et al. Determination of stilbenes (trans-astringin, cis- and trans-piceid, and cis- and trans-resveratrol) in Portuguese wines. J Agric Food Chem 1999; 47: 2666-2670.

24 Wang SY, Chen CT, Wang CY, et al. Resveratrol content in strawberry fruit is affected by preharvest conditions. J Agric Food Chem 2007; 55: 8269-8274.

25 Ragab AS, Van FJ, Jankowski B, et al. Detection and quantitation of resveratrol in tomato fruit (Lycopersicon esculentum Mill.). J Agric Food Chem 2006; 54: 7175-7179.

26 Counet C, Callemien D, Collin S. Chocolate and cocoa: new sources of trans-resveratrol and trans-piceid. Food Chemistry 2006; 98: 649-657.

27 Zamora-Ros R, Andres-Lacueva C, Lamuela-Raventos RM, et al. Concentrations of resveratrol and derivatives in foods and estimation of dietary intake in a Spanish population: European Prospective Investigation into Cancer and Nutrition (EPIC)-Spain cohort. Br J Nutr 2007: 1-9.

28 Renaud S, de Lorgeril M. Wine, alcohol, platelets, and the French paradox for coronary heart disease. Lancet 1992; 339: 1523-1526.

29 Artaud-Wild SM, Connor SL, Sexton G, et al. Differences in coronary mortality can be explained by differences in cholesterol and saturated fat intakes in 40 countries but not in France and Finland: a paradox. Circulation 1993; 88: 2771-2779.

30 Opie LH, Lecour S. The red wine hypothesis: from concepts to protective signalling molecules. Eur Heart J 2007; 28: 1683-1693.

31 Sin DD, Wu L, Man SFP. The relationship between reduced lung function and cardiovascular mortality: a population-based study and a systematic review of the literature. Chest 2005; 127: 1952-1959.

32 Dudley JI, Lekli I, Mukherjee S, et al. Does white wine qualify for French paradox? Comparison of the cardioprotective effects of red and white wines and their constituents: resveratrol, tyrosol, and hydroxytyrosol. J Agric Food Chem 2008; 56: 9362-9373.

33 Tabak C, Arts IC, Smit HA, et al. Chronic obstructive pulmonary disease and intake of catechins, flavonols, and flavones: the MORGEN Study. Am J Respir Crit Care Med 2001; 164: 61-64.

34 Hertog M, Hollman P, van de Putte B. Content of potentially anticarcinogenic flavonoids of tea infusions, wines, and fruit juices. J Agric Food Chem 1993; 41: 1242-1246.

35 Jerkovic V, Callemien D, Collin S. Determination of stilbenes in hop pellets from different cultivars. J Agric Food Chem 2005; 53 : 4202-4206.

36 Bak I, Lekli I, Juhasz B, et al. Cardioprotective mechanisms of Prunus cerasus (sour cherry) seed extract against ischemiareperfusion-induced damage in isolated rat hearts. Am J Physiol Heart Circ Physiol 2006; 291: H1329-H1336.

37 Ocké MC, Bueno-de-Mesquita HB, Goddijn HE, et al. The Dutch EPIC food frequency questionnaire. I. Description of the questionnaire, and relative validity and reproducibility for food groups. Int J Epidemiol 1997; 26: Suppl. 1, S37-S48.

38 Ocké MC, Bueno-de-Mesquita HB, Pols MA, et al. The Dutch EPIC food frequency questionnaire. II. Relative validity and reproducibility for nutrients. Int J Epidmiol 1997; 26: Suppl. 1, S49-S58.

39 Goldberg DM, Yan J, Ng E, et al. A global survey of trans-resveratrol concentrations in commercial wines. Am J Enol Vitic 1995; 46: 159-165.

40 Moreno-Labanda JF, Mallavia R, Perez-Fons L, et al. Determination of piceid and resveratrol in Spanish wines deriving from Monastrell (Vitis vinifera L.) grape variety. J Agric Food Chem 2004; 52: 5396-5403. 